\title{
Application of network link prediction in drug discovery
}

\author{
Khushnood Abbas ${ }^{1,2^{*}}$, Alireza Abbasi², Shi Dong ${ }^{1}$, Ling Niu', Laihang Yu', Bolun Chen ${ }^{4}$, Shi-Min Cai ${ }^{3}$ and \\ Qambar Hasan ${ }^{5}$
}

\author{
${ }^{*}$ Correspondence: \\ abbas@cigit.ac.cn \\ ${ }^{2}$ School of Engineering \\ and Information Technology, \\ University of New South \\ Wales, Canberra, NSW 2006, \\ Australia \\ Full list of author information \\ is available at the end of the \\ article
}

\begin{abstract}
Background: Technological and research advances have produced large volumes of biomedical data. When represented as a network (graph), these data become useful for modeling entities and interactions in biological and similar complex systems. In the field of network biology and network medicine, there is a particular interest in predicting results from drug-drug, drug-disease, and protein-protein interactions to advance the speed of drug discovery. Existing data and modern computational methods allow to identify potentially beneficial and harmful interactions, and therefore, narrow drug trials ahead of actual clinical trials. Such automated data-driven investigation relies on machine learning techniques. However, traditional machine learning approaches require extensive preprocessing of the data that makes them impractical for large datasets. This study presents wide range of machine learning methods for predicting outcomes from biomedical interactions and evaluates the performance of the traditional methods with more recent network-based approaches.
\end{abstract}

Results: We applied a wide range of 32 different network-based machine learning models to five commonly available biomedical datasets, and evaluated their performance based on three important evaluations metrics namely AUROC, AUPR, and F1-score. We achieved this by converting link prediction problem as binary classification problem. In order to achieve this we have considered the existing links as positive example and randomly sampled negative examples from non-existant set. After experimental evaluation we found that Prone, $A C T$ and $L R W_{5}$ are the top 3 best performers on all five datasets.

Conclusions: This work presents a comparative evaluation of network-based machine learning algorithms for predicting network links, with applications in the prediction of drug-target and drug-drug interactions, and applied well known network-based machine learning methods. Our work is helpful in guiding researchers in the appropriate selection of machine learning methods for pharmaceutical tasks.

Keywords: Data-driven drug discovery, Network link prediction, Poly-pharmacy, Polypharmacy side effects prediction, Drug-target prediction author(s) and the source, provide a link to the Creative Commons licence, and indicate if changes were made. The images or other third party material in this article are included in the article's Creative Commons licence, unless indicated otherwise in a credit line to the material. If material is not included in the article's Creative Commons licence and your intended use is not permitted by statutory regulation or exceeds the permitted use, you will need to obtain permission directly from the copyright holder. To view a copy of this licence, visit http:// creativecommons.org/licenses/by/4.0/. The Creative Commons Public Domain Dedication waiver (http://creativecommons.org/publi cdomain/zero/1.0/) applies to the data made available in this article, unless otherwise stated in a credit line to the data. 


\section{Background}

Diseases are a complex set of phenomena that include non-linear relationships between an individual cell and an organism [1]. To find the proper response against a particular disease, i.e. designing and developing appropriate drugs, requires consideration of many such phenomena [2]. The traditional drug discovery processe is expensive involving five steps [3-8]: (1) discovery and pre-clinical research; (2) safety review; (3) clinical research; (4) regulatory review (e.g., by the American Food and Drug Administration, FDA); and (5) regulatory post-market safety monitoring. That makes it time-consuming [5] and costly [9]. However, data-driven computerised drug discovery methods offers the potential to speed up the drug discovery process. There are currently four categories of methods for data-driven drug discovery: (1) ligandbased approaches; (2) docking approaches; (3) network-based approaches; and (4) machine learning-based approaches. Ligand-based target prediction assumes that similar drugs tend to bind similar targets (e.g. diseases). As this approach utilises the similarity of the ligands for making prediction, it requires examples of interactions between drugs and targets for prediction. Docking-based methods make predictions based on the three-dimensional structure of proteins, and is greatly limited when the structure of the protein is unknown. However, network-based and machine learningbased approaches attempt to overcome the aforementioned limitations of the other two approaches [10]. Nevertheless, not only ligand- and docking-based prediction approaches require prior data, but also network- and machine-learning approaches depend on reliable prior data (training data for ML, relevant interaction data for network approaches).

Here, we formulate a data-driven drug discovery approach that models drug-target interactions (DTI) as networks between two sets of nodes: the drug candidates, and the entities affected by the drugs (i.e. diseases, genes, and other drugs), which are referred to as targets. Our aim is to predict the missing nodes (i.e. drugs and targets) and links between them. For example, we attempt to predict which candidate drugs might treat a list of diseases. There is a large amount of data identifying which drugs treats which diseases, but some diseases have very few drugs available. Thus, discovering which existing drugs can treat them is of great importance. Further, it is critical to determine which drugs have side effects in the presence of other drugs as interactions among drugs may be harmful or lethal to patients. Therefore, drug interaction networks are considered to predict what is the likelihood of reactions between combinations of drugs in a patient's body. Likewise, we formulate a drug-target interaction network to predict missing links between drugs and target diseases.

When considering $n$ drugs, then there will be $n *(n-1) / 2$ combinations of drugdrug relationships for trials. Because a patient could be taking more than two medicines together, the resulting combinations are of an even higher-order and are not feasible to test via experiments. Thus, link prediction offers an important solution. Besides, it allows to find additional uses of existing drugs, with 30\% of 84 drugs introduced in 2013 being reused. Many drugs affect more than one particular protein or gene, and some medical conditions involve multiple genes and proteins. Modeling such situations as network interactions and formulating a link prediction problem enables drug-target gene prediction. 


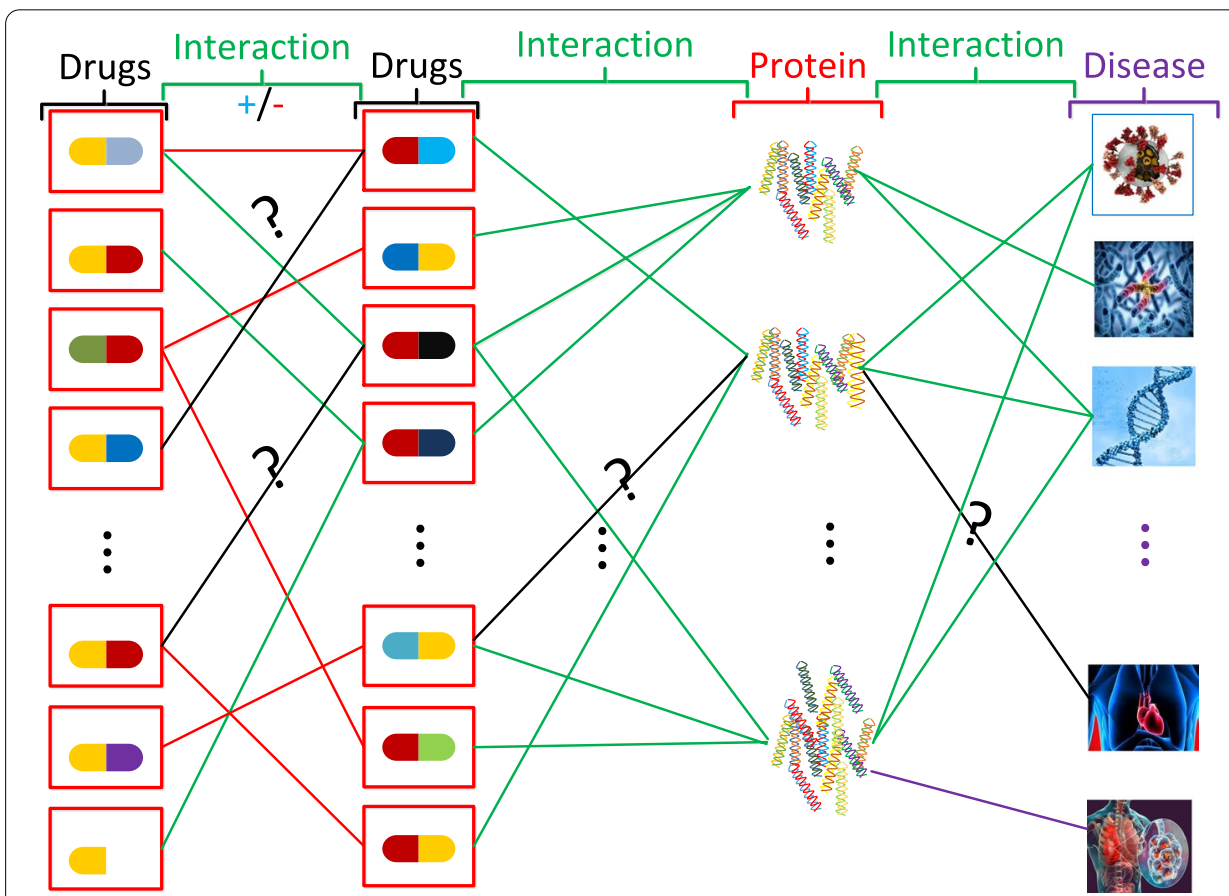

Fig. 1 A depiction of the link prediction approach in drug discovery. This is a heterogeneous drug-target and drug-drug interaction network

Traditional machine learning approaches applied to the drug-target interaction (DTI) problem have many constraints, including dimensionality (for complex and large pharmacological datasets) and incompleteness [11], sparsity, and heterogeneity (mainly in biological datasets). For instance, logistic regression and support vector machines suffer from the high dimensionality and numerous implicit relationships in the data. These are the result of many factors including measurement technologies [12] and bias problems during the recording of the data [13]. Besides, the spreading speed of diseases or other causes of infection such as viruses evolve quickly is not considered in traditional machine learning methods. In addition, the hierarchical nature of biological data (connections among genes, proteins, and so on) cannot be easily modeled by traditional machine learning approaches. Therefore, there is a need for methods and models capable of addressing these problems.

Network-based approaches are gaining attention because of their simplicity (node and edge representation) which effectively considers high dimensionality and heterogeneity as well as implicit relationships. For drug discovery, these relationships include sharing a common chemical formula and structure or affecting the same protein. Such ability supports reusing existing drugs in new ways, as with a recent breast cancer treatment [14]. As noted previously, this accelerates the drug discovery process, saving time and expense [15]. As an example, other medications such as Duloxetine, used for treating depression, have been found powerful in treating urine leakage issues [16]. Thus, we can 
consider drug discovery as a missing link problem between chemicals and proteins as shown in Fig. 1.

This work exploits network-based link prediction models for solving the following pharmaceutical problems:

- Drug-target interaction prediction: This task is to predict which drug will affect which protein this is one of the application in drug repurposing.

- Drug-drug side effect prediction: From existing drug-drug side effect data, we can create a network, in which a link reflects the two drugs (nodes) has shown some side effects. So in this task we predict which new drug combinations can cause side effect.

- Disease-gene association prediction: Some disease affects the genes which is more lethal as it can transfer to the next generations. Therefore in this task we aim to predict which new disease can affect which particular gene.

- Disease-drug association prediction: Some drugs might not be pharmaceutical chemicals such as arsenic. So in this problem, we aim to predict which drug is associated to which disease.

We also evaluate the performance of the models using five publicly available pharmacological datasets, and report the performance of these models according to three different evaluation metrics.

\section{Network-based approaches for drug discovery}

Researchers have also explored network topology-based link prediction methods for drug-target interaction (DTI) prediction. Pech et al. [17] propose a sparse learning method for link prediction. Fokoue et al. [18] propose a knowledge graph DTI prediction framework called Tiresias. Chen et al. [19] presented Network-based Random Walk with Restart on the Heterogeneous network (NRWRH), based on random walk with restart on a heterogeneous network, by constructing the drug similarity, protein similarity, and drug-target network as a heterogenous network. Cheng et al. and Huang et al. [20, 21] solve the DTI problem using a bipartite network and proposed three DTI prediction methods: drug-based similarity inference (DBSI), target-based similarity inference (TBSI), and network-based inference (NBI). The drug side effect similarity inference (DSESI) method [22] utilises the drug-drug chemical similarity and the phenotypic side effect similarity. The multiple target optimal intervention (MTOI) method [23] solves this problem with two steps: finding the known drug-target links, and applying a multiple target control inference mechanism. Luo et al. [24] combine various attributes from heterogeneous networks and propose a novel network integration pipeline, DTINet for DTI prediction. This solution utilises drug and protein distributions in each network and embeds the high dimensional protein and drug data into lower dimensions. Another proposal suggests a meta-path-based methodology to separate the semantic highlights of DTIs from heterogeneous networks [25].

Figure 1 shows how a drug discovery problem can be converted to a link prediction problem. The relationship network is heterogeneous as many entities are related, such as drug-drug, drug-gene, drug-disease, disease-gene, and drug-drug side effects (see 
Fig. 1). However, we consider only monopartite and bipartite individual networks in our study.

\section{Deep learning based approaches for drug discovery}

Duvenaud et al. [26] present a deep learning model for generating molecular features based on convolutional neural networks. Gilmer et al. [27] propose a deep learning framework using a message passing neural network for molecular property prediction. You et al. [28] propose a Reinforcement Learning-based Graph Convolutional Policy Network (GCPN) as a goal-directed graph generation model. This approach is highly applicable to both chemistry and drug discovery where the goal is to find new molecules with given molecular properties such as drug similarity and synthetic accessibility. Cao et al. [29] propose a Generative Adversarial Network (GAN) generative approach that supports creating molecules with desired molecular properties. Coley et al. [30] and Kearnes et al. [31] solve molecular graph representation problems by applying a graph convolutional network to an undirected molecular graph. Along with molecular graph structural attributes, they also consider other factors such as atom and bond attributes, neighbouring atoms, and radii. Xie et al. [32] propose a Crystal Graph Convolutional Neural Network framework that is able to learn material properties from the crystal atomic link structure, which can be very helpful in new material design. Ktena et al. [33] use graph convolutional neural networks for graph similarity prediction to identity brain disorders. Parisot et al. [34, 35] use a graph convolutional network for brain disease prediction. Assouel et al. [36] propose a conditional graph generative model.

\section{Genomic and phenotypic study for drug discovery}

Advances in the study of genomes have generated huge volumes of genomic and transcriptomic data, including a diverse set of disease samples, standard tissue samples, and cell lines. Gene expression data from these studies have been widely adopted for research purposes. One of the widely adopted genomic datasets is the Library of Integrated Network-based Cellular Signatures (LINCS) [37] that contains extensive data from cancer cell lines treated under different conditions. One benefit of using genomic data is 'signature reversion' that enables the study of reverse relationships as well, i.e., drug-disease and disease-drug. The other area of study of computational drug discovery is phenotypic information, in which a study of the phenome is performed to identify the genetic association with disease [38]. This is also known as a phenome-wide association study. For example, Bisgin et al. [39] use phenotypic information from the Side Effect Resource (SIDER) database [40] and applied Dirichlet Allocation Model for drug re-positioning discovery. Phenotype information can be used to make other kinds of association predictions also. For example, some researchers used phenotypic link prediction between drug-gene and phenotype-disease information [41].

\section{Role of drug chemical structure in drug discovery}

Another computational method for drug discovery is examination of the chemical structure of drugs by representing the molecular structure as a network. Hence, this approach is based on the assumption that compounds with similar structures will act similarly against the same proteins. Other methods make use of the molecular structures 
themselves, as with 2D topological fingerprints and 3D informatics. Researchers such as Swamidass et al. [42] study which chemical structures modulate which disease relevant phenomes. This helps predict what other drugs will affect the same protein or disease. Tan et al. [43] employ chemical structures along with semantic gene similarity to construct a drug similarity network, which can be then used to find novel drug-target relations. Further, Ng. et al. [44] propose ligand Enrichment of Network Topological Similarity (ligENTS) to identify novel drug-target relations by using the chemical structure of the drug for drug repurposing task.

\section{Study of drug combinations in drug discovery}

Another area of work is drug combination prediction as many diseases are the results of complex events involving many complex molecular structures. Predicting interactions between multiple molecules and processes is thus important. In many cases, more than one drug is used to treat diseases, such as diabetes, cancer, and bacterial infections, as the drug combinations are found to be more effective than single drug therapies $[45,46]$. For example, B-cell lymphoma (DLBCL) is a malignant cancer requiring multiple targeted drugs, some or all of which are administered at one time. However, the presence of one drug sometimes increases or decreases the effects of other drugs. Combinations can even cause fatalities from Adverse Drug Reactions (ADRs), which are the fourth leading cause of death in the United States [47]. Such interactions are usually not observed during clinical trials of individual drugs as testing of combinations in vivo is both time consuming and expensive.

Therefore, the problem of drug combinations can be formulated as two types of the link prediction problem, namely treating disease, and side effects and reactions. In Figure 1 shows these links with red and green colors, respectively. Drug-drug side effect prediction is an important task in its own right, but some researchers like Campillos et al. [22] use drug side effects as features for predicting novel target prediction. Further, Zitnik et al. [48] propose Dacagon, a graph convolutional network-based framework, to predict which drug combinations cause which side effects in patients. Li et al. [49] develop a bipartite drug-target network to find similar drugs using a graph node similarity approach. Li et al. [50] develop a multilayered network of gene-disease and drug-target network to identify new therapeutic uses of existing drugs. Wu et al. [51] formulate a drug-disease heterogeneous network to identify similar drug and disease pairs. Jin et al. [52] develop a novel method to identify similar drugs for cancer by exploiting off-target effects which act on important cancer cell signalling pathways. This approach employs a model called the Bayesian Factor Regression Model (BFRM), introduces a new network component called Cancer Signalling Bridges (CSB), and integrates the two into a hybrid method called CSB-BFRM. The researchers have applied this approach to breast and prostate cancer cells.

\section{Methodology and data}

\section{Problem description: the drug discovery problem}

We investigate the drug discovery problem by applying various contemporary models to different pharmacological situations to see how well the prediction models are able 
to facilitate drug discovery process. To carry out our task, we first build the networks, either mono-partite or bipartite, and ensured they were undirected. We convert bipartite datasets into the corresponding biadjacency matrix, and then apply the link prediction models to solve the following drug discovery problems:

1. Drug-Target prediction: The aim is to predict which drug will affect which unknown proteins, considering the bipartite networks of drugs and their target proteins.

2. Drug-Disease prediction: The aim is to study drug chemical structures and target proteins (as the disease and drugs both affects proteins) to find similarities between drug structures. It has been found that similar drug structures affect similar proteins. We represent each chemical structure as a network. Once a similar drug is found, it can be used to target similar proteins. There is currently a lack of systematic research in this area.

3. Drug-Drug reaction prediction: This problem examines the search for combinations of drugs for conditions that require targeting more than one protein, as with degenerative neurological conditions, such as Alzeheimer's and Parkinson's. We incorporate known combinations that cause adverse side effects (headaches, vomiting, rashes, etc.) to predict which additional combinations might cause reactions in patients.

4. Disease-Gene association prediction: Thanks to high-throughput screening technologies, we have large volumes of genomic data. Yet, there are many diseases for which a genomic basis is unknown. Genomic alleles and malignant mutations are continuously sequenced, which is why most of them are identified or annotated. Traditionally, linkage analysis has been done to find non-experimental disease-gene associations, and it has been based on the likelihood of observing alleles. However, this kind of analysis fails for a multifactorial and heterogeneous diseases. Considering genomic data association is a newer approach to solve this problem, but with the downside of producing hundreds of candidates for complex diseases, which hinders experimental validation. Therefore, we present a network approach for genomic data analysis. In addition to existing network approaches, such as common neighbour, path-based and random walk-based methods, recent developments in network-based learning technologies, such as geometric deep learning, offer the prospect of using genomic data to find gene-disease associations that are still unknown.

\section{Link prediction models for drug discovery}

Given a set of nodes $V$ and a set of edges $E$, the corresponding network is $G(V, E)$ at time $t$. The graph $G$ can be effectively represented by a $(|\mathrm{v}| \times|\mathrm{v}|)$ adjacency matrix $A$ where the entry $A_{i j}=w$ is non-zero if there is a link between node $i$ and $j$, and 0 otherwise. Many problems in drug discovery can be modelled as mono-partite undirected networks (e.g., drug-drug networks), but some such as drug-target networks require bi-partite models, i.e. having two different sets of nodes: drugs and targets. In this case, the link relationship can be represented as a $B_{r \times k}$ biadjacency network [53] whose two parts have $r$ and $k$ number of nodes. The corresponding adjacency matrix can then be represented as the biadjacency matrix [54] 


$$
A=\left[\begin{array}{cc}
0 & B \\
B^{T} & 0
\end{array}\right]
$$

The link prediction problem is then defined as predicting the unobserved link between two nodes during the time interval $t+\Delta t$. Most of the existing methods solve the link prediction problem by calculating a likelihood score between two nodes $\left(S_{i j}\right)$. To validate the models' performance, we randomly select links from the test set, $E^{T}=E-E^{P}$ and use the remaining set as a training set. The training and test sets are mutually exclusive, i.e., $E^{T} \cap E^{P}=\Phi$.

In this section, we present the link prediction techniques for drug discovery. Our initial steps included the use of existing models meant to solve problems in other domains like social networks. The various models we considered, both old and new, are as follows:

1. Common Neighbours $(\mathrm{CN})$ : Two nodes are more likely to be connected if they have more common neighbours. If $\Gamma(i)$ represents the vector associated with node $i$ that includes the neighbours of node $i$, we express this relationship as [55]

$$
S_{i j}=|\Gamma(i) \cap \Gamma(j)|
$$

2. Salton index (Cosine similarity): This is another measure of commonality that measures the cosine of the angle between two vectors of the adjacency matrix, corresponding to given nodes $i$ and $j$. It is calculated as below:

$$
S_{i j}=\frac{|\Gamma(i) \cap \Gamma(j)|}{\sqrt{k_{i} * k_{j}}} .
$$

where $k_{i}$ is the total degree of node $i$ [56].

3. Jaccard index [57]: This method from the early 20th century is the proportion of common neighbours between two nodes $i$ and $j$ in the total number of neigbours

$$
S_{i j}=\frac{|\Gamma(i) \cap \Gamma(j)|}{|\Gamma(i) \cup \Gamma(j)|}
$$

4. Sorensen index [58]: This index was developed especially for ecological community data and is defined as

$$
S_{i j}=\frac{2 \times|\Gamma(i) \cap \Gamma(j)|}{k_{i}+k_{j}} .
$$

5. Hub Promoted Index (HPI) [59]: This index was developed for considering metabolic networks. Under this model, links adjacent to hub nodes (high degree nodes) are assigned a high score, as the denominator depends on the minimum of the degrees of the two nodes. It is defined as

$$
S_{i j}=\frac{|\Gamma(i) \cap \Gamma(j)|}{\operatorname{mink}_{i}, k_{j}} .
$$

6. Hub Depressed Index (HDI) [60]: This index is similar to the Sorensen index, but it also considers the measurement of the opposite effect: 


$$
S_{i j}=\frac{|\Gamma(i) \cap \Gamma(j)|}{\max \left\{k_{i}, k_{j}\right\}}
$$

7. Leicht-Holme-Newman Index (LHN-I) [11]: This model assigns a high score to common neighbour nodes while penalizing according to the degree of each node:

$$
S_{i j}=\frac{|\Gamma(i) \cap \Gamma(j)|}{k_{i} \cdot k_{j}} .
$$

8. Preferential Attachment (PA) [61]: This is based on the assumption that nodes with higher links will form more links. Therefore, the PA-based link prediction model is simply a product of the degree of the two nodes:

$$
S_{i j}=k_{i} \cdot k_{j}
$$

9. Adamic-Adar (AA) [62]: AA uses the assumption that less-connected nodes should be given more weight for future link prediction:

$$
S_{i j}=\sum_{z \in \Gamma(i) \cap \Gamma(j)} \frac{1}{\log k_{z}}
$$

10. Resource Allocation Index (RA)[63]: This is inspired by the resource allocation process, and measures how much resource is transmitted between th etwo nodes $i$ and $j$ :

$$
S_{i j}=\sum_{z \in \Gamma(i) \cap \Gamma(j)} \frac{1}{k_{z}}
$$

11. Local Path Index (LP) $[64,65]$ : This index considers the local paths between the two nodes considering neighbours of second order, which makes it fairly inexpensive to compute. For the adjacency matrix $A$ of the network, the LP-based score is

$$
S_{i j}^{L P}=A^{2}+\in A^{3}
$$

where $\epsilon$ is a free parameter and when 0 , the LP index is equal to $C N$. $\left(A^{n}\right)_{i j}$ representing the number of paths of length 3 between nodes $i$ and $j$.

12. Katz global path indicator [66]: Theis index considers the number of paths, their lengths, and their weights (shorter paths counting more heavily), which is computed as:

$$
S_{i j}=\sum_{l=1}^{\infty} \beta^{l}\left|p a t h s_{i j}^{l}\right|=\beta A_{i j}+\beta^{2}\left(A^{2}\right)_{i j}+\beta^{3}\left(A^{3}\right)_{i j} . .=(I-\beta A)^{-1}-I
$$

where paths $s_{i j}^{l}$ is set of all paths with lenght $l$ which connects node $i$ and node $j, \beta$ is the weight attenuation factor. In order to ensure the convergence of the series, the value of $\beta$ must be less than the reciprocal of the largest eigenvalue of the adjacency matrix $A$.

13. Average Commute Time (ACT) [67]: This metric determines closeness by commute time. The smaller the average commute time between the two nodes, the closer the 
nodes are. It considers the average number of steps required by a random walker starting from $i$ to reach $j$ and vice versa. It can be calculated as:

$$
S_{i j}^{A C T}=\frac{1}{l_{i i}^{+}+l_{j j}^{+}-2 l_{i j}^{+}} \quad \begin{aligned}
& v^{i}=\sqrt{\Lambda} \cdot U^{T} \vec{e}_{i} \\
& l_{i j}^{+}=v_{i}^{T} v_{j}
\end{aligned}
$$

Where $l_{i j}^{+}$entry of Laplacian matrix $L=D-A$. Where $D$ is the degree matrix.

14. Cosine Similarity Based on Random Walk (Cos+) [68]: This method uses the inner product. Letting $e^{i}$ be the $N \times 1$ vector with the $i$ th entry $=1$, then

$$
v^{i}=\sqrt{\Lambda} \cdot U^{T} \vec{e}_{i}
$$

If $U$ is the orthonormal matrix made of eigenvectors of the Laplacian matrix $L, \Lambda=$ $\operatorname{diag}\left(\lambda_{i}\right)$, and

$$
S_{i j}^{\cos +}=\cos (i, j)^{+}=\frac{l_{i j}^{+}}{\sqrt{l_{i i}^{+} l_{j j}^{+}}} .
$$

where symbols have usual meaning.

15. Random Walk with Restart (RWR)[69]: This is inspired by the Google PageRank algorithm. Suppose a random walker at node $i$ takes a random step towards any of the neighbours of $i$ with probability $\alpha$. Therefore the probability of returning to node $i$ again is $(1-\alpha)$. Thus, the probability that the random walker reaches node $j$ can be given as $\vec{P}_{i}=\alpha L^{T} \vec{P}+\left(1-\alpha L^{T}\right) e^{i}$, where $L^{T}$ is the transition matrix $\mathrm{L}_{\mathrm{ij}}=\frac{1}{\mathrm{k}_{\mathrm{i}}}$ if node $i$ and $j$ are connected other wise $L_{i j}=0$.

$$
S_{i j}^{R W R}=P_{i j}+P_{j i}
$$

where $p_{i j}$ is the $j$ th element of vector $p_{i}$.

16. Local Random Walk (LRW) [70]: A random walker starts at node $i$ and reaches node $j$ within some number of random steps. The initial density vector is $\overrightarrow{\Pi_{(0)}}=\overrightarrow{e_{i}}$. The LRW similarity index at any time $t$ can be formulated as

$$
S_{i j}^{L R W}(t)=P_{i} \Pi_{i j}(t)+P_{j} \Pi_{j i}(t),
$$

where $P$ is the initial configuration function.

17. SimRank (SimR) [71]: This is a general similarity measure that considers two nodes are similar if their neighbours are similar (connected to similar nodes):

$$
s_{i j}^{\operatorname{SimR}}=C \frac{\sum_{z \in \Gamma(i)} \sum_{z^{\prime} \in \Gamma(j)} S_{z z^{\prime}}^{\operatorname{SimR} R}}{k_{i} k_{j}} .
$$

18. CN based on Transferring Similarity (TSCN) [72]: This method uses the $\mathrm{CN}$ index but considers the transference similarity deined as

$$
S_{i j}^{T r}=\varepsilon \sum_{v} S_{i j}^{C N} S_{v j}^{T r}+S_{i j}^{C N},
$$

where $S_{v j}^{T r}$ is the transferring similarity. 
19. Superimposed Local Random Walk Indicator (SRW) [70]: This method is based on LRW, summing the $t$ step and its previous results to obtain the value of SRW:

$$
S_{i j}^{S R W}(t)=\sum_{l=1}^{t} S_{i j}^{L R W}(l)=q_{i} \sum^{t} \pi_{i j}(l)+q_{j} \sum_{l=1}^{t} \pi_{j i}(l) .
$$

20. Local Naive Bayes form of CN (LNBCN) [73]:

$$
S_{i j}^{L N B R A}=\sum_{w \in \Gamma(i) \cap \Gamma(j)} \frac{1}{k_{w}}\left(\log R_{w}+\log S\right)
$$

where $S=\frac{P\left(A_{0}\right)}{P\left(A_{1}\right)}$, and $A_{0}$ and $A_{1}$ are the connection and disconnection variables, respectively. $R_{W}$ is the role function of node $w$.

21. Local Naive Bayes form of RA (LNBRA) [59]: This method is based on Naive Bayes and formulated as follows

$$
S_{i j}^{L N B R A}=|\Gamma(i) \cap \Gamma(j)| \log s+\sum_{w \in \Gamma(i) \cap \Gamma(j)} \log R_{w},
$$

where $S=\frac{P\left(A_{0}\right)}{P\left(A_{1}\right)}$, and $A_{0}$ and $A_{1}$ are the connection and disconnection variables, respectively. $R_{W}$ is the role function of node $w$.

22. Leicht-Holme-Newman (LHN2) Index [11]: This index is based on the assumption that two nodes are similar if their neighbours are also similar. It is an extension of the Katz index. The LHN2 likelihood score can be given as

$$
S_{i j}^{L H N 2}=2 m \lambda_{1} D^{-1}\left(I-\frac{\Phi A}{\lambda_{1}}\right)^{-1} D^{-1}
$$

where $D$ is the degree matrix with $D_{i j}=\lambda_{i j} k_{i}$, and $\Phi \varepsilon(0,1)$ is the free parameter.

23. Cosine based on $\mathrm{L}+$ (CosPlus): This similarity measure is based on the inner product measure and cosine similarity between node vectors $i$ and $j$. It is given as

$$
S_{i j}^{\operatorname{Cos}+}=\frac{V_{i}^{T} \cdot V_{i}^{T}}{\left|V_{i}\right| \cdot\left|V_{j}\right|}
$$

24. Matrix Forest Index (MFI) [74]: The MFI index similarity between nodes $i$ and $j$ can be given as ratio of the number of spanning rooted forests so that nodes $i$ and $j$ belong to the same tree rooted at $i$ to all spanning rooted forests. This similarity index is expressed as

$$
S_{i j}^{M F I}=(I+L)^{-1} .
$$

25. Prone [75]: This method first initialises the embedding by sparse matrix factorization and further uses spectral analysis for local and global structural information of the node.

26. DeepWalk [76]: This model learns node low dimensional embeddings based on random walks. It has two hyper parameters: the walk length $l$ and the window size $w$.

27. Node2vec [77]: Node2vec is an application of the Word2vec model for graphs [78]. Word2vec is a state-of-the-art framework for word embedding. Based on similar 
skip-gram concept Node2vec works on neighbourhood nodes and generates low dimensional embeddings. Node2vec can be generalised according to need, such as if one wants to embed similarity based on distance or based on role of the node in network.

28. LINE [79]: This model generates low-level node embeddings considering first order and second order of the nodes' similarity. Further, this model samples based on edge weight, improving performance for large scale networks. It is special case of DeepWalk when the size of the vertices' context is kept at 1.

29. NetMF [80]: Similar to DeepWalk and Line, this method also employs the skip-gram technique for low dimensional embedding. In fact, this model unifies the LINE, PTE [81], DeepWalk, Node2vec, and the proposed matrix factorization framework.

30. High-Order Proximity-preserved Embedding (HOPE) [82]: This method draws from PageRank and the Katz index and uses singular value decomposition for making low rank approximations.

31. NetSMF [80]: Network Embedding as Sparse Matrix Factorization (NetSMF) is based on spectral sparsification, and is an improved extension of NetMF. It is costly for large networks as it requires a large number of random walks.

32. GraRep [83]: Grarep depends on singular value decomposition. It uses nodes' cooccurrence information by exponentiating the matrix with different powers, making it unsuitable for large graphs.

\section{Model performance evaluation metrics}

We convert the link prediction problem to a binary classification problem by using a positive class from the test set $\left(E^{T}\right)$. Further, we generate negative samples for training and test sets. To sample negative links for training and test data set, we assume all the testing links were known, and thus sample negative train links only from other unknown links. This enables us to evaluate the accuracy of link prediction methods based on binary classification evaluation metrics. To evaluate performance we used three standard machine learning metrics as follows:

Precision Precision measures the proportion of true positives against all positives. For $T_{P}$ items predicted correctly as positive and $F_{P}$ are predicted incorrectly as positive (i.e., false positives). Precision is calculated as:

$$
\text { Precision }=\frac{T_{P}}{T_{P}+F_{P}} .
$$

To measure the misclassification of actual positives, we use the Recall metric, penalising the score with false negatives. If $F_{N}$ is the number of false negatives, then recall is defined as

$$
\text { Recall }=\frac{T_{P}}{T_{P}+F_{N}} .
$$

Finally, a combined scoring mechanism called the F1-Score is a harmonic mean between precision and recall. It is also known as the True Positive Rate (TPR): 


$$
F 1-\text { score }=\frac{\text { Precision } * \text { Recall }}{(\text { Precision }+ \text { Recall })}
$$

The False Positive Rate (FPR) is calculated as

$$
F P R=\frac{F_{P}}{\left(T_{N}+F_{P}\right)},
$$

where $F_{P}$ is the number of false positives, and $T_{N}$ is the number of true negatives.

AUROC The Area Under the Receiver Operating Characteristics (AUROC) value is the area under the plot between True Positive Rate (TPR) and the False Positive Rate (FPR). It represents the trade-off between TP and FP prediction rates. The TPR is also known as sensitivity, recall, or probability of detection. AUROC measures the separability of the classifier and is therefore a vital metric.

$A U P R$ The area under the Precision and Recall (AUPR) curve estimates the combined accuracy of precision and recall simultaneously. In other words, precision-recall pair points are obtained by considering different threshold values. This measure estimates the efficiency in the presence of unbalanced classes and indicates the models' ability to cope with skewed distributions.

\section{Datasets used}

We used several publicly available datasets for pharmacological problems:

- Disease-Gene Association network (DGA): This is a disease-gene association network dataset from the Stanford SNAP group and contains disease and gene association information [84].

- Drug-Disease Association network (DDA): There are two kinds of nodes in this network: drugs and diseases. Some nodes in the drugs class are non-pharmaceutical chemicals such as arsenic. Diseases include skin disease and myocardial infarction. These interactions predict which drug treats which diseases.

- Disease-Target Interaction network (DTI): This source is from the Stanford SNAP online data repository with DTI information similar to MATADOR. We use the protein and chemical identifiers as the two kinds of nodes in a bipartite network [85].

- MATADOR database: This is a manually annotated drug and target database freely available [86] and containing 15,843 Drug-Target interactions (DTIs). The original data set contains 13 fields, but in this study we only used two as a sample: chemical identifier, and protein identifier.

Table 1 Some properties of the data sets used in our experiments

\begin{tabular}{llccc}
\hline Dataset & $\mid$ V & $|E|$ & From nodes & To nodes \\
\hline MATADOR & 3702 & 15,843 & 801 & 2901 \\
DTI & 3932 & 18,690 & 284 & 3648 \\
DDI & 1514 & 48,514 & 1514 & 1514 \\
DGA & 7813 & 21,357 & 519 & 729 \\
DDA & 7197 & 466,656 & 5535 & 1662 \\
\hline
\end{tabular}


- Drug-Drug Interaction network (DDI): This source is also from the Stanford SNAP group, containing information about drug-drug interactions approved by the United States Food and Drug Administration. It is a mono-partite network as compared to the previous bipartite datasets. A drug-drug relationship is formed when the pharmacological effect of one drug is affected by another drug [85].

The Table 1 summarises the contents of the datasets.

\section{Analysis and results}

As cross-validation is a standard technique to test the generalizing ability of models or algorithms, we performed a 10-fold cross-validation. We randomly selected a percentage of edges and removed them from the network, and used those removed edges as test data in each model. Using these two sets, we then evaluated the performance of the models. Table 2 shows the results, where the best results are highlighted in each case. For several models we considered a variation of hyper-parameters which gives better results such as random walk length etc. For instance, the numbers after LRW model name (i.e. $L R W_{3}, L R W_{4}, L R W_{5}$ ) reflects the number of random-walk steps. We make the following points in our own analysis:

- On the Disease-Gene associate (DGA) dataset, the Average Commute Time (ACT) model achieved the best AUROC score, the $L R W_{3}$ (Local Random Walk) model with 3 steps achieved the best AUPR score, and the LHN2 (Leicht-Holme-Newman) with parameter 0.95 achieved the best F1-score. All the three models are considering global similarity measures.

- On the Drug-Disease association (DDA) dataset, again the ACT achieved the best AUROC score, LRW with 5 steps achieved the best AUPR score, and LHN2 with parameter 0.95 had the best F1-score.

- On the Disease-Target Interaction (DTI) dataset, NetMF performed the best on all three metric scores.

- On the MATADOR dataset, NetMF performed best on all three metrics.

- On the Drug-Drug Interaction (DDI) dataset, as the only mono-partite network, the Prone model performed best on all three metrics.

Overall, the models that performed best on the five benchmark datasets were Prone, $A C T$ and $L R W_{5}$ are the top 3. The Freidman test results are presented in Table 3.

\section{Advatanges and disadvantages of these methods}

No doubt the graphs are state-of-the art tools being utilised to solve problems of complex systems. But as they give strength to model any real world problem they also have weaknesses specially in our case. As graphs are representations of nodes and edges. A node alone carries less or no information at all. The node of the graph carries more information if it is the part of bigger networks or graphs. Consequently our model will perform worse for node/s which are either alone or connected to small subgraphs. For example in case of drug-drug side effect prediction if we have more examples of side-effect interaction examples with other drugs then the unknown interaction can be 


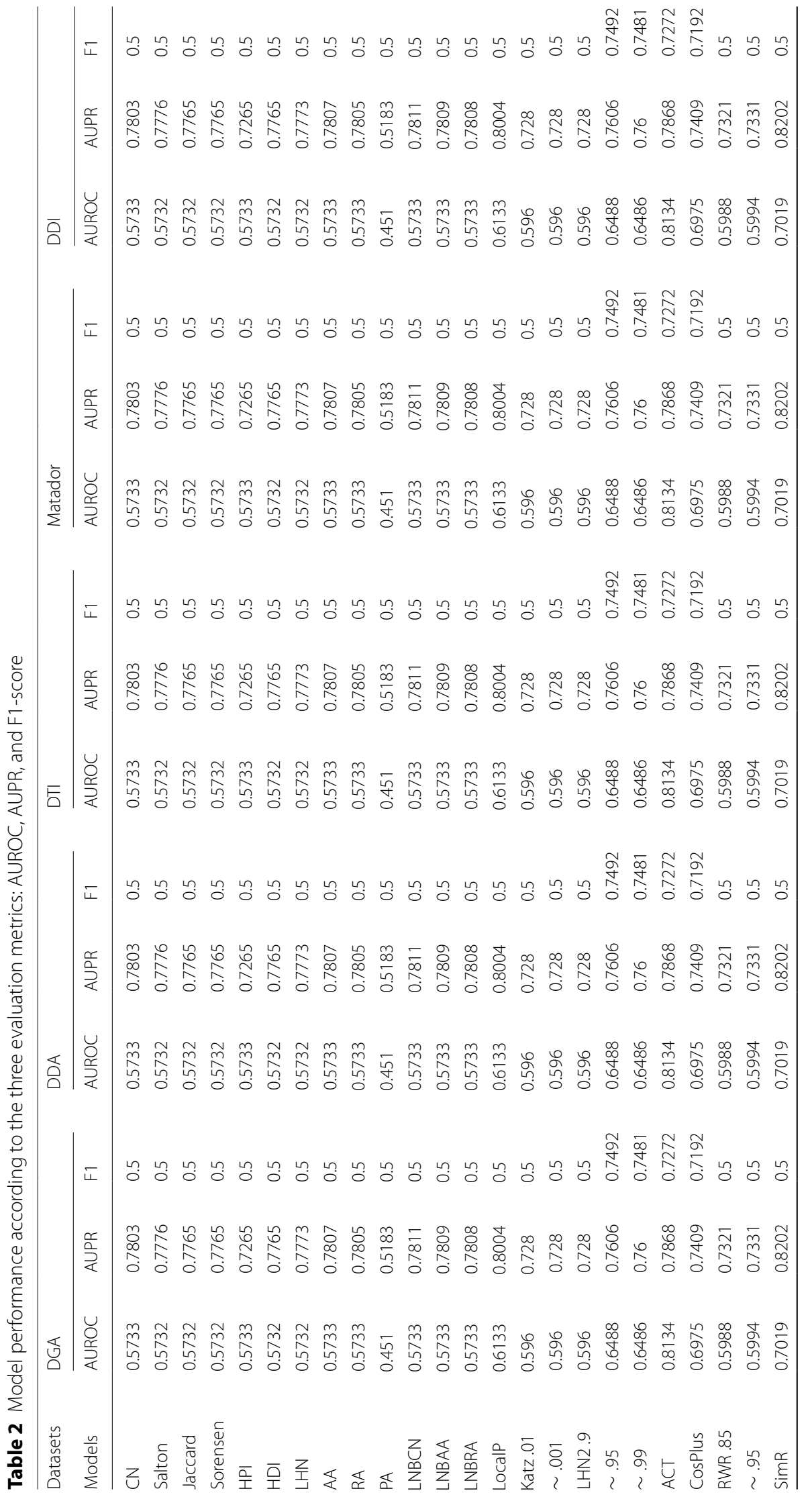




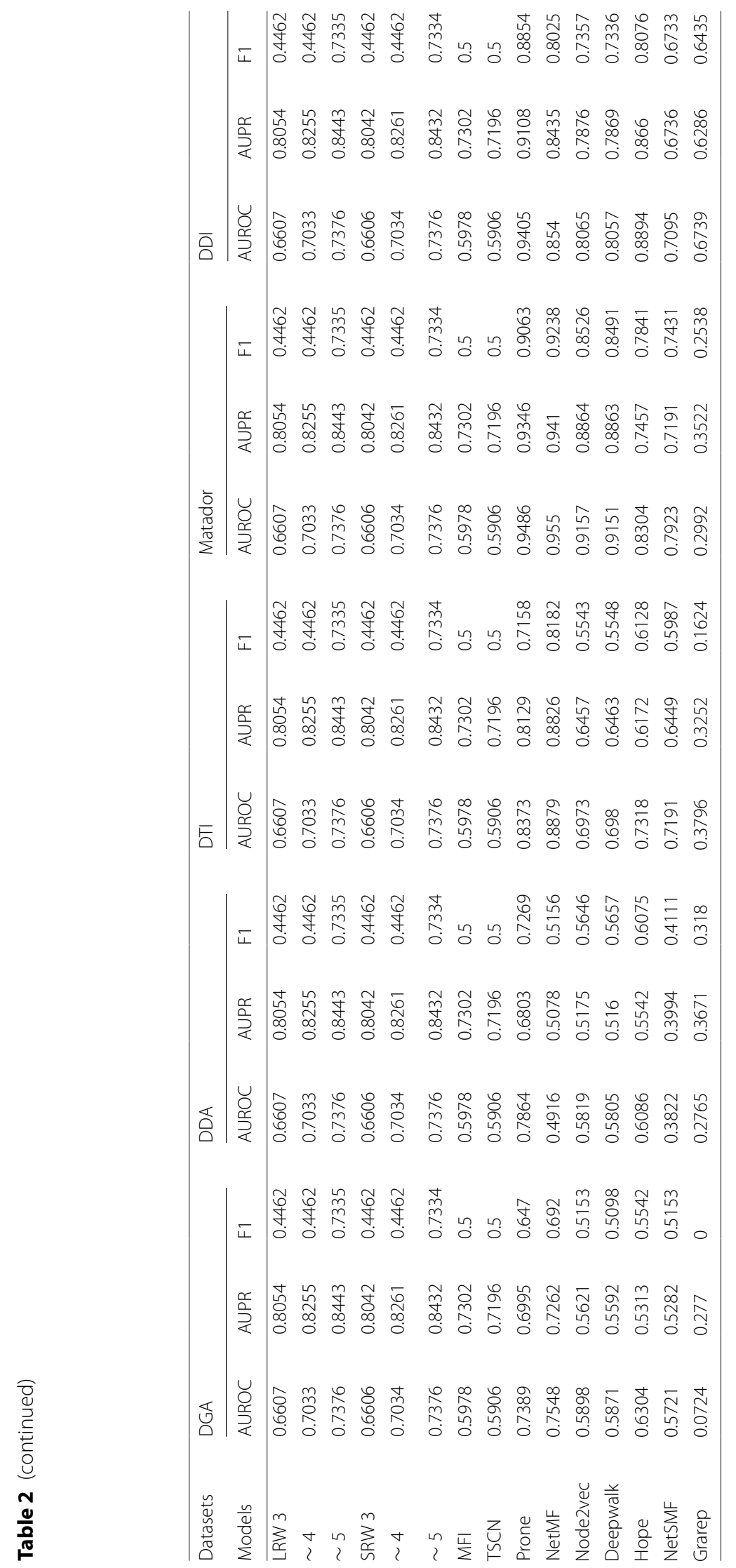


Table 3 Average performance of all models across datasets

\begin{tabular}{lllllllllll}
\hline Model & CN & Salton & Jaccard & Sorens & HPI & HDI & LHN & AA & RA & PA \\
\hline Mean rank & 28.6 & 34.6 & 34.6 & 34.6 & 28.6 & 34.6 & 34.6 & 28.6 & 28.6 & 38 \\
Model & LNBCN & LNBAA & LNBRA & LocalP & Katz.01 & $\sim .001$ & LHNII.9 & $\sim .95$ & $\sim .99$ & ACT \\
Mean rank & 28.6 & 28.6 & 28.6 & 16.6 & 21.8 & 21.8 & 21.8 & 14.4 & 15.4 & 3 \\
Model & CosPlus & RWR.85 & $\sim .95$ & SimR & LRW_3 & $\sim$ _4 & $\sim$ _5 & SRW_3 & $\sim$ _4 & $\sim$ _5 \\
Mean rank & 11 & 18.8 & 17.8 & 9.8 & 12.4 & 8.8 & 5.7 & 13.4 & 7.8 & 5.7 \\
Model & MFI & TSCN & prone & netmf & node2vec & Deepwalk & hope & netsmf & grarep & \\
Mean rank & 19.8 & 23.8 & 2 & 8.6 & 13.2 & 13.6 & 8.2 & 19.6 & 34 & \\
\hline
\end{tabular}

predicted with better accuracy. The same logic goes with drug-target interaction prediction and so on. Consequently we can say the more edges we have the better the predictibility of a model. So network based methods will fail to predict interaction for novel node/s which has no prior interaction say it is new drug or new target.

\section{Statistical test}

After running the Friedman test [87] for model comparison we have found the following mean ranking of models' across the datasets. To achieve this we have only considered one evaluation metric i.e. AUROC and converted into error using formula $A U R O C_{\text {error }}=1-A U R O C$. According to Table 3 analysis we can say on an average Prone, $A C T$ and $L R W_{5}$ are the top 3 best performers over all five data-sets. The p-value is found $1.11022 E-16$. Rest of the tests we have reported in Additional file 1.

\section{Conclusion and discussion}

This study highlighted the need for utilising data-driven approaches for enhancing drug discovery processes particularly using the drug-target interactions (DTI) forming biomedical networks. This allows us to utilise network-based techniques and in particular link prediction approaches to predict the interactions (links), or missing links, between drugs and their targets including diseases, proteins or other drugs. This approach has been already shown promising outcomes in reusing existing drugs for treating breast cancer [14], or identifying a new drug for urine leakage issues treatment [16]. We further discussed more advanced network-based approaches are required to addresses the existing challenges in using traditional machine learning approaches such as data related issues (e.g. dimensionality, incompleteness, sparsity, heterogeneity, and the hierarchical structure), and incapability of consideration of the spreading speed of diseases.

In this work, we have compared several state-of-the-art link prediction models on five different drug-related data-sets modeling drug-disease, drug-drug, drug-gene, and drug-target interactions to see the implications for drug discovery. We compared the results of the models using three evaluation metrics namely AUROC, AUPR, and F1-score. The results indicate that Prone, $A C T$ and $L R W_{5}$ are the top 3 best performers on all five data-sets. These models are important as they only need prior link or relationship information, which avoids the cost of feature engineering. The statistical models are effective particularly on graphs as the graph is basically a non-Euclidean data representation. Traditional machine learning tools were intended for Euclidean data sets, 
thus explaining the performance. There are excellent opportunities for future work to represent and solve network-based biological and pharmaceutical problems using stateof-the-art deep learning techniques.

\title{
Methodological limitations
}

In our analysis we have considered positive links only from examples we had in the data-set. Rest of the space is considered as negative links which is acceptable for mathematical algorithmic perspective. This techniques is well utilised by machine learning community. The negative link space is all the unknowns for which we don't have any examples. So one of the limitations for our methodology is that if we have lesser number of examples the algorithmic predictive ability will be negatively affected. In other words the more examples we have the better the predictability of the algorithm will be.

\begin{abstract}
Abbreviations
AUROC: The Area Under the Receiver Operating Characteristics curve; AUPR: The area under the Precision and Recall curve; TPR: True positive rate; FPR: False positive rate; FDA: Food and Drug Administration; ML: Machine learning; DTI: Drug-target interactions; TBSI: Target based similarity inference; NBI: Network based inference; NRWRH: Network-based Random Walk with Restart on the Heterogeneous network; DSESI: Drug side effect similarity inference; MTOI: Multiple target optimal intervention; GCPN: Graph Convolutional Policy Network; GAN: Generative Adversarial Network; LINCS: Library of integrated network-based cellular signatures; SIDER: Side effect resource; ligENTS: Ligand Enrichment of Network Topological Similarity; DLBCL: Diffuse large B-cell lymphoma; ADR: Adverse Drug Reactions; BFRM: Bayesian Factor Regression Model; CSB: Cancer Signalling Bridges; CN: Common Neighbours; HPI: Hub Promoted Index; HDI: Hub Depressed Index; LHNI: Leicht-Holme-Newman Index; PA: Preferential Attachment; AA: Adamic-Adar; RAI: Resource Allocation Index; LPI: Local Path Index; ACT: Average Commute Time; RWR: Random Walk with Restart; RWRn: Random Walk with Restart with n steps; LRW: Local Random Walk; LRWn: Local Random Walk with n steps; TSCN: CN based on Transferring Similarity; SLRWI: Superimposed Local Random Walk Indicator; LNBCN: Local Naive Bayes form of CN; LNBRA: Local Naive Bayes form of RA; LHN2: Leicht-Holme-Newman; MFI: Matrix Forest Index; LINE: Large-scale Information Network Embedding; HOPE: High-Order Proximity-preserved Embedding; NetSMP: Network Embedding as Sparse Matrix Factorization; Prone: A graph vertex representation model name; GraRep: A graph vertex representation model name; DeepWalk: A graph vertex representation model name; Node2Vec: A graph vertex representation model name; DGA: Disease-Gene Association network; DDA: Drug-Disease Association network; DTI: Disease-Target Interaction network; DDI: Drug-Drug Interaction network; MATADOR: Protein-chemical interactions.
\end{abstract}

\section{Supplementary Information}

The online version supplementary material available at https://doi.org/10.1186/s12859-021-04082-y.

Additional file 1: Data normality test

\section{Acknowledgements}

This work was supported in part by the Key Scientific and Technological Research Projects in Henan Province under Grant 202102210379 and also by Zhoukou Normal University super scientific project grant ZKNUC2018019. This research was also supported in part by the Chinese National Natural Science Foundation under Grant No. 61602202, the Natural Science Foundation of Jiangsu Province under contracts BK20160428, the Six talent peaks project in Jiangsu Province under contract XYDXX-034 and the project in Jiangsu Association for science and technology.

\section{Authors' contributions}

$K A, A A, D S$ conceptualised the solution to the problem. KA performed the experiment and reported the results. $L Y, C B$, $\mathrm{SC}, \mathrm{NL}$, and $\mathrm{HQ}$ helped in writing the manuscripts. Further NL, DS and KA analysed the results. All authors have read and approved the manuscript.

\section{Funding}

This work was supported in part by the Key Scientific and Technological Research Projects in Henan Province under Grant 202102210379 and also by Zhoukou Normal University super scientific project Grant ZKNUC2018019. This research was also supported in part by the Chinese National Natural Science Foundation under Grant No. 61602202, the Natural Science Foundation of Jiangsu Province under contracts BK20160428, the Six talent peaks project in Jiangsu Province under contract XYDXX-034 and the project in Jiangsu Association for science and technology. The funding body did not influence the study, collection, analysis or interpretation of any data.

Availability of data and materials

The data-sets can be found at https://github.com/khushnood/DataDrivenDrugDiscovery/tree/master. 


\section{Declarations}

Ethics approval and consent to participate

Not applicable.

\section{Consent for publication}

Not applicable.

\section{Competing interests}

The authors declare that they have no competing interests.

\section{Author details}

${ }^{1}$ School of Computer Science and Technology, Zhoukou Normal University, Zhoukou 466001, China. ${ }^{2}$ School of Engineering and Information Technology, University of New South Wales, Canberra, NSW 2006, Australia. ${ }^{3}$ School of Computer Science and Engineering, University of Electronic Science and Technology of China, Chengdu 610054, China. ${ }^{4}$ College of Computer and Software Engineering, Huaiyin Institute of Technology, Huaian 223003, China. ${ }^{5}$ Centre for Cellular and Molecular Biology, School of Life and Environmental Science, Deakin University, Burwood, VIC 3125, Australia.

Received: 15 August 2020 Accepted: 16 March 2021

Published online: 12 April 2021

\section{References}

1. Csermely P, Korcsmáros T, Kiss HJ, London G, Nussinov R. Structure and dynamics of molecular networks: a novel paradigm of drug discovery: a comprehensive review. Pharmacol Ther. 2013;138(3):333-408.

2. Loscalzo J, Barabasi A-L. Systems biology and the future of medicine. Wiley Interdiscip Rev Syst Biol Med. 2011;3(6):619-27.

3. Scannell JW, Blanckley A, Boldon H, Warrington B. Diagnosing the decline in pharmaceutical R\&D efficiency. Nat Rev Drug Discov. 2012;11(3):191.

4. Kola I, Landis J. Can the pharmaceutical industry reduce attrition rates? Nat Rev Drug Discov. 2004;3(8):711.

5. Paul SM, Mytelka DS, Dunwiddie CT, Persinger CC, Munos BH, Lindborg SR, Schacht AL. How to improve R\&D productivity: the pharmaceutical industry's grand challenge. Nat Rev Drug Discov. 2010;9(3):203.

6. Gov, U. FDA drug developement process. 2019. https://www.fda.gov/patients/learn-about-drug-and-device-approvals/ drug-development-process. Accessed on 10/31/2019.

7. Gov, U. FDA drug developement process. 2019. https://www.fda.gov/drugs/drug-information-consumers/fdas-drugreview-process-ensuring-drugs-are-safe-and-effective. Accessed on 10/31/2019.

8. Gov, U. FDA drug developement process. 2019. https://www.fda.gov/drugs/development-approval-process-drugs. Accessed on 10/31/2019.

9. Xue H, Li J, Xie H, Wang Y. Review of drug repositioning approaches and resources. Int J Biol Sci. 2018;14(10):1232.

10. Zhou L, Li Z, Yang J, Tian G, Liu F, Wen H, Peng L, Chen M, Xiang J, Peng L. Revealing drug-target interactions with computational models and algorithms. Molecules. 2019;24(9):1714.

11. Leicht EA, Holme P, Newman ME. Vertex similarity in networks. Phys Rev E. 2006;73(2):026120.

12. Žitnik M, Zupan B. Data imputation in epistatic maps by network-guided matrix completion. J Comput Biol. 2015:22(6):595-608.

13. Wang P, Xu B, Wu Y, Zhou X. Link prediction in social networks: the state-of-the-art. Sci China Inf Sci. 2015;58(1):1-38.

14. You J, McLeod RD, Hu P. Predicting drug-target interaction network using deep learning model. Comput Biol Chem. 2019;80:90-101.

15. Ashburn T, Thor KB. Drug repositioning: identifying and developing new uses for existing drugs. Nat Rev Drug Discov. 2004;3(8):673.

16. Wang S-M, Lee H-K, Kweon Y-S, Lee CT, Lee K-U. Overactive bladder successfully treated with duloxetine in a female adolescent. Clin Psychopharmacol Neurosci. 2015;13(2):212

17. Pech R, Hao D, Po M, Zhou T. Predicting drug-target interactions via sparse learning. Google Scholar. 2017.

18. Fokoue A, Sadoghi M, Hassanzadeh O, Zhang P. Predicting drug-drug interactions through large-scale similarity-based link prediction. In: European semantic web conference. Springer; 2016. p. 774-89.

19. Chen X, Liu M-X, Yan G-Y. Drug-target interaction prediction by random walk on the heterogeneous network. Mol BioSyst. 2012:8(7):1970-8.

20. Cheng F, Liu C, Jiang J, Lu W, Li W, Liu G, Zhou W, Huang J, Tang Y. Prediction of drug-target interactions and drug repositioning via network-based inference. PLoS Comput Biol. 2012;8(5):e1002503.

21. Huang H, Zhang P, Qu XA, Sanseau P, Yang L. Systematic prediction of drug combinations based on clinical side-effects. Sci Rep. 2014;4:7160

22. Campillos M, Kuhn M, Gavin A-C, Jensen $\sqcup$, Bork P. Drug target identification using side-effect similarity. Science. 2008;321(5886):263-6.

23. Yang K, Bai H, Ouyang Q, Lai L, Tang C. Finding multiple target optimal intervention in disease-related molecular network. Mol Syst Biol. 2008;4(1):228.

24. Luo Y, Zhao X, Zhou J, Yang J, Zhang Y, Kuang W, Peng J, Chen L, Zeng J. A network integration approach for drug-target interaction prediction and computational drug repositioning from heterogeneous information. Nat Commun. 2017:8(1):1-13.

25. Fu G, Ding Y, Seal A, Chen B, Sun Y, Bolton E. Predicting drug target interactions using meta-path-based semantic network analysis. BMC Bioinform. 2016;17(1):160.

26. Duvenaud DK, Maclaurin D, Iparraguirre J, Bombarell R, Hirzel T, Aspuru-Guzik A, Adams RP. Convolutional networks on graphs for learning molecular fingerprints. In: Advances in neural information processing systems. 2015. p. 2224-32. 
27. Gilmer J, Schoenholz SS, Riley PF, Vinyals O, Dahl GE. Neural message passing for quantum chemistry. In: Proceedings of the 34th international conference on machine learning, vol. 70. 2017. p. 1263-72. JMLR. org.

28. You J, Liu B, Ying Z, Pande V, Leskovec J. Graph convolutional policy network for goal-directed molecular graph generation. In: Advances in neural information processing systems. 2018. p. 6410-21.

29. De Cao N, KipfT. Molgan: an implicit generative model for small molecular graphs. 2018. arXiv preprint arXiv:1805.11973

30. Coley CW, Barzilay R, Green WH, Jaakkola TS, Jensen KF. Convolutional embedding of attributed molecular graphs for physical property prediction. J Chem Inf Model. 2017;57(8):1757-72.

31. Kearnes S, McCloskey K, Berndl M, Pande V, Riley P. Molecular graph convolutions: moving beyond fingerprints. J Comput Aided Mol Des. 2016;30(8):595-608

32. Xie T, Grossman JC. Crystal graph convolutional neural networks for an accurate and interpretable prediction of material properties. Phys Rev Lett. 2018;120(14):145301.

33. Ktena SI, Parisot S, Ferrante E, Rajchl M, Lee M, Glocker B, Rueckert D. Distance metric learning using graph convolutional networks: application to functional brain networks. In: International conference on medical image computing and computer-assisted intervention. Springer; 2017. p. 469-77.

34. Parisot S, Ktena SI, Ferrante E, Lee M, Moreno RG, Glocker B, Rueckert D. Spectral graph convolutions for population-based disease prediction. In: International conference on medical image computing and computer-assisted intervention. Springer; 2017. p. 177-85.

35. Parisot S, Ktena SI, Ferrante E, Lee M, Guerrero R, Glocker B, Rueckert D. Disease prediction using graph convolutional networks: application to autism spectrum disorder and Alzheimer's disease. Med Image Anal. 2018;48:117-30.

36. Assouel R, Ahmed M, Segler MH, Saffari A, Bengio Y. Defactor: differentiable edge factorization-based probabilistic graph generation. 2018. arXiv preprint arXiv:1811.09766.

37. Vidović D, Koleti A, Schürer SC. Large-scale integration of small molecule-induced genome-wide transcriptional responses, kinome-wide binding affinities and cell-growth inhibition profiles reveal global trends characterizing systemslevel drug action. Front Genet. 2014;5:342.

38. Hebbring SJ. The challenges, advantages and future of phenome-wide association studies. Immunology. 2014;141(2):157-65.

39. Bisgin H, Liu Z, Fang H, Kelly R, Xu X, Tong W. A phenome-guided drug repositioning through a latent variable model. BMC Bioinform. 2014;15(1):267.

40. Kuhn M, Campillos M, Letunic I, Jensen $\sqcup$, Bork P. A side effect resource to capture phenotypic effects of drugs. Mol Syst Biol. 2010;6(1):343.

41. Hoehndorf R, Oellrich A, Rebholz-Schuhmann D, Schofield PN, Gkoutos GV. Linking pharmgkb to phenotype studies and animal models of disease for drug repurposing. In: Biocomputing 2012. World Scientific; 2012. p. 388-99.

42. Swamidass SJ. Mining small-molecule screens to repurpose drugs. Brief Bioinform. 2011;12(4):327-35.

43. Tan F, Yang R, Xu X, Chen X, Wang Y, Ma H, Liu X, Wu X, Chen Y, Liu L, et al. Drug repositioning by applying 'expression profiles' generated by integrating chemical structure similarity and gene semantic similarity. Mol BioSyst. 2014;10(5):1126-38.

44. Ng C, Hauptman R, Zhang Y, Bourne PE, Xie L. Anti-infectious drug repurposing using an integrated chemical genomics and structural systems biology approach. In: Biocomputing 2014. World Scientific; 2014. p. 136-47.

45. Webster RM. Combination therapies in oncology. Nat Rev Drug Discov. 2016;15(2):81.

46. Lehár J, Krueger AS, Avery W, Heilbut AM, Johansen LM, Price ER, Rickles RJ, Short lii GF, Staunton JE, Jin X, et al. Synergistic drug combinations tend to improve therapeutically relevant selectivity. Nat Biotechnol. 2009;27(7):659-66.

47. Flockhart D, Honig P, Yasuda S, Rosebraugh C. Preventable adverse drug reactions: a focus on drug interactions. Centers for Education and Research on Therapeutics; 2009. p. 452.

48. Zitnik M, Agrawal M, Leskovec J. Modeling polypharmacy side effects with graph convolutional networks. Bioinformatics. 2018:34(13):457-66.

49. Li J, Lu Z. A new method for computational drug repositioning using drug pairwise similarity. In: 2012 IEEE international conference on bioinformatics and biomedicine, 2012. IEEE. p. 1-4.

50. Li J, Lu Z. Pathway-based drug repositioning using causal inference. BMC Bioinform. 2013;14(16):3.

51. Wu C, Gudivada RC, Aronow BJ, Jegga AG. Computational drug repositioning through heterogeneous network clustering. BMC Syst Biol. 2013;7(5):6.

52. Jin G, Fu C, Zhao H, Cui K, Chang J, Wong ST. A novel method of transcriptional response analysis to facilitate drug repositioning for cancer therapy. Cancer Res. 2012;72(1):33-44.

53. Godsil C, Royle GF. Algebraic graph theory, vol. 207. Springer; 2013.

54. Lu Y. Link prediction in drug-target interactions network using similarity indices. PhD thesis, University of Cambridge; 2015.

55. Newman ME. Clustering and preferential attachment in growing networks. Phys Rev E. 2001;64(2):025102.

56. Chowdhury GG. Introduction to modern information retrieval. Facet Publishing: 2010.

57. Jaccard P. Étude comparative de la distribution florale dans une portion des alpes et des jura. Bull Soc Vaudoise Sci Nat. 1901;37:547-79.

58. Sorensen TA. A method of establishing groups of equal amplitude in plant sociology based on similarity of species content and its application to analyses of the vegetation on Danish commons. Biol Skar. 1948;5:1-34.

59. Ravasz E, Somera AL, Mongru DA, Oltvai ZN, Barabási A-L. Hierarchical organization of modularity in metabolic networks. Science. 2002;297(5586):1551-5.

60. Lü L, Zhou T. Link prediction in complex networks: a survey. Phys A. 2011;390(6):1150-70.

61. Barabási A-L, Albert R. Emergence of scaling in random networks. Science. 1999;286(5439):509-12.

62. Adamic LA, Adar E. Friends and neighbors on the web. Soc Netw. 2003;25(3):211-30.

63. Zhou T, Lü L, Zhang Y-C. Predicting missing links via local information. Eur Phys J B. 2009;71(4):623-30.

64. Lü L, Jin C-H, Zhou T. Similarity index based on local paths for link prediction of complex networks. Phys Rev E. 2009;80(4):046122.

65. Al Hasan M, Zaki MJ. A survey of link prediction in social networks. In: Social network data analytics. Springer; 2011. p. 243-75.

66. Katz L. A new status index derived from sociometric analysis. Psychometrika. 1953;18(1):39-43.

67. Klein DJ, Randić M. Resistance distance. J Math Chem. 1993;12(1):81-95. 
68. Fouss F, Pirotte A, Renders J-M, Saerens M. Random-walk computation of similarities between nodes of a graph with application to collaborative recommendation. IEEE Trans Knowl Data Eng. 2007;19(3):355-69.

69. Brin S, Page L. The anatomy of a large-scale hypertextual web search engine. Comput Netw ISDN Syst. 1998;30(1-7):107-17.

70. Liu W, Lü L. Link prediction based on local random walk. EPL (Europhys Lett). 2010;89(5):58007.

71. Jeh G, Widom J. Simrank: a measure of structural-context similarity. In: Proceedings of the eighth ACM SIGKDD international conference on knowledge discovery and data mining. ACM; 2002. p. 538-43.

72. Sun D, Zhou T, Liu J-G, Liu R-R, Jia C-X, Wang B-H. Information filtering based on transferring similarity. Phys Rev E. 2009:80(1):017101.

73. Liu Z, Zhang Q-M, Lü L, Zhou T. Link prediction in complex networks: a local naïve bayes model. EPL (Europhys Lett). 2011;96(4):48007.

74. Chebotarev P, Shamis E. The matrix-forest theorem and measuring relations in small social groups. 2006. arXiv preprint arXiv:math/0602070

75. Zhang J, Dong Y, Wang Y, Tang J, Ding M. Prone: fast and scalable network representation learning. In: Proceedings of the twenty-eighth international joint conference on artificial intelligence, IJCAl-19. International joint conferences on artificial intelligence organization 2019. p. 4278-84. https://doi.org/10.24963/ijcai.2019/594.

76. Perozzi B, Al-Rfou R, Skiena S. Deepwalk: Online learning of social representations. In: Proceedings of the 20th ACM SIGKDD international conference on knowledge discovery and data mining. ACM; 2014. p. 701-710.

77. Grover A, Leskovec J. node2vec: Scalable feature learning for networks. In: Proceedings of the 22nd ACM SIGKDD international conference on knowledge discovery and data mining. ACM; 2016. p. 855-864.

78. Mikolov T, Chen K, Corrado G, Dean J. Efficient estimation of word representations in vector space. 2013. arXiv preprint arXiv:1301.3781.

79. Tang J, Qu M, Wang M, Zhang M, Yan J, Mei Q. Line: Large-scale information network embedding. In: Proceedings of the 24th international conference on World Wide Web. International World Wide Web Conferences Steering Committee; 2015. p. 1067-77.

80. Qiu J, Dong Y, Ma H, Li J, Wang K, Tang J. Network embedding as matrix factorization: Unifying deepwalk, line, pte, and node2vec. In: Proceedings of the eleventh ACM international conference on web search and data mining. ACM; 2018. p. 459-67.

81. Tang J, Qu M, Mei Q. Pte: Predictive text embedding through large-scale heterogeneous text networks. In: Proceedings of the 21th ACM SIGKDD international conference on knowledge discovery and data mining. ACM; 2015. p. 1165-74.

82. Ou M, Cui P, Pei J, Zhang Z, Zhu W. Asymmetric transitivity preserving graph embedding. In: Proceedings of the 22nd ACM SIGKDD international conference on knowledge discovery and data mining. ACM; 2016. p. 1105-14.

83. Cao, S., Lu, W., Xu, Q: Grarep: learning graph representations with global structural information. In: Proceedings of the 24th ACM international on conference on information and knowledge management. ACM; 2015. p. 891-900.

84. Davis AP, Grondin CJ, Johnson RJ, Sciaky D, King BL, McMorran R, Wiegers J, Wiegers TC, Mattingly CJ. The comparative toxicogenomics database: update 2017. Nucleic Acids Res. 2017;45(D1):972-8.

85. Wishart DS, Feunang YD, Guo AC, Lo EJ, Marcu A, Grant JR, Sajed T, Johnson D, Li C, Sayeeda Z, et al. Drugbank 5.0: a major update to the drugbank database for 2018. Nucleic Acids Res. 2017;46(D1):1074-82.

86. Günther S, Kuhn M, Dunkel M, Campillos M, Senger C, Petsalaki E, Ahmed J, Urdiales EG, Gewiess A, Jensen $\perp$, et al. Supertarget and matador: resources for exploring drug-target relationships. Nucleic Acids Res. 2007;36(suppl-1):919-22.

87. Friedman M. The use of ranks to avoid the assumption of normality implicit in the analysis of variance. J Am Stat Assoc. 1937;32(200):675-701.

\section{Publisher's note}

Springer Nature remains neutral with regard to jurisdictional claims in published maps and institutional affiliations.

Ready to submit your research? Choose BMC and benefit from:

- fast, convenient online submission

- thorough peer review by experienced researchers in your field

- rapid publication on acceptance

- support for research data, including large and complex data types

- gold Open Access which fosters wider collaboration and increased citations

- maximum visibility for your research: over 100M website views per year

At BMC, research is always in progress.

Learn more biomedcentral.com/submissions 